International Journal of Advanced Biological and Biomedical Research Available online at http:www.ijabbr.com

Volume 7, Issue 4 (2019) pp. 342-352

DOI:10.33945/SAMI/IJABBR.2019.4.6

Original Article

\title{
Bioorganic Investigation of Encapsulated Cysteine Derivative into Polymeric Nanocarrier
}

Ehab M. Ali*, Ahmed Solaiman Hamed

Department of Chemistry, University of Fallujah, Iraq

*Corresponding Author E-mail: ehab201725@yahoo.com

Received: 03 May 2019, Revised: 15 June 2019, Accepted: 18 June 2019

\begin{abstract}
In this work, the copolymer-based synthesized Cysteine-loaded nanocarriers were prepared by a routine protocol, coprecipitation method. It is the first report to investigate the neuroprotective potential and biocompatibility of Cysteine derivatives loaded into poly(ethylene glycol)-block-poly( $\varepsilon$-caprolactone) methyl ether (PEG-b-PCL). The average size of the polymeric/empty NCs was $89 \mathrm{~nm}$ and for polymeric/Synthesized derivative of Cysteine was $126 \mathrm{~nm}$. The Drug Loading efficiency was 81\%. The concentration of Polymeric NCs was $2.1 \times 10^{10}$ particles/mL and the zeta potential of polymeric/empty and polymeric/Synthesized derivative of Cysteine NCs $-5 \mathrm{mV}$ and $-11 \mathrm{mV}$, respectively. Biological part of this work was investigated in the SH-SY5Y human neuroblastoma cell line using cell viability and toxicity assays. The concentration of polymeric NCs below $1 \times 10^{10}$ particles/mL was described as a zero-point damageable for the cell line. Also, the Synthesized derivative of Cysteine encapsulated into polymeric NCs has more neuroprotective effect as compared to free Cysteine at lower concentration, and therefore, has a significant neuroprotective potential against Z-VAD-fmk and St-evoked SH-SY5Y cell damage.
\end{abstract}

Key words: Biocompatibility, Neuroprotective, Polymer, Biopolymer.

\section{Introduction}

Urbanization expansion, using radio-based services and rising average age of the world's population, has become the major factor to increase central nervous system (CNS) disorders. During the last decade, investigating biological systems based on biochemical mechanism of neuronal cell damage and biological models has been highlighted and considered by the researchers. (Ghasemi et al., 2018; Nasseri et al., 2018; Rabiee et al., 2019b; Rabiee et al., 2019c; Rabiee \& Rabiee, 2019; Uttara et al., 2009; Vafajoo et al., 2018) It is vividly depicted that many inclusive diseases such as Parkinson, Huntington and Alzheimer crop up as a result of neurodegenerative processes, in addition the root mechanism of these diseases remotely related to the mitochondrial dysfunction and disrupted neuronal intracellular that cause calcium homeostasis which in turn leads to unusual activation of $\mu$-calpain. As a matter of fact, the inhibition of hyper activity of calpain could lead to inhibition of calcium homeostasis and prevention of neuroprotective diseases 
(Carragher, 2006; Deljoo et al., 2019; Rabiee et al., 2019a; Rabiee et al., 2019; Szczęch et al., 2017). Without a shadow of a doubt, the effectiveness of the neuroprotective substances must take step progress, but it should be noted that the choice of these substances is very sensitive due to their chemical limitations which lead to peripheral toxicity and side effects (Svenson, 2012). In addition, the other issue which is remotely related to treatment of neurodegeneration is the incompetent delivery of drugs by the Blood-Brain Barrier (BBB). The central nervous system could be protected by biological membrane whose competent transport of chemical drugs and upholding the stability of the environment through BloodBrain Barrier have become a great challenge.

Needless to say during the last decade, countless efforts have been made to implement prominent evolution in nano-scale generation of drugs in the diagnosis and treatment of diseases. In this regard, most scientists have focused on micro- and nanocarriers as drug delivery systems for these inclusive diseases such as Parkinson, Huntington and Alzheimer. In comparison to the regular and extracted-based natural drugs, especially coupled with nano-systems, it could be obvious to understand hopeful opportunities nano-systems would bring, i.e. improving in vivo bioavailability, boosting the efficiency of insoluble and mid soluble compounds. Moreover, reducing of side effects has been shown and of course they could be functionalized by chemical agents for targeting delivery (Chen et al., 2016).

Recently, S-Allyl-L-Cysteine (PMI-CYSTEINE) (Figure 1) has introduced an agent of suppression of $\mu$ - and $m$-calpain activities followed by a bright promising neuroprotective compound (Imai et al., 2014; Karimi et al., 2018a; Karimi et al., 2018b; Rabiee \& Rabiee, 2018a, Rabiee \& Rabiee 2018b). Previously, the PMI-CYSTEINE showed some crucial biological effects such as anticancer, (Thomson \& Ali, 2003) antihepatotoxic(Kodai et al., 2007) and neurotrophic activity in cultured rat hippocampal neurons (Moriguchi et al., 1997). The result showed that PMI-CYSTEINE suppressed the TM-induced degradation of full length $\alpha$-spectrin and remarkable suppression of $\mu$ - and $m$-calpain activities at higher concentrations.

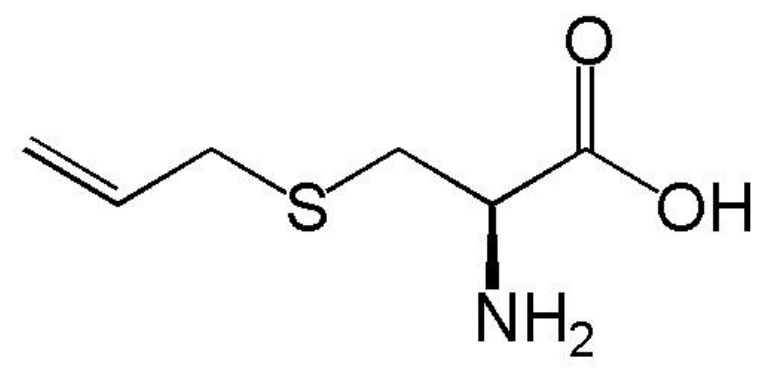

Figure 1. S-Allyl-L-Cysteine (PMI-CYSTEINE)

In this work, we have been focused on using Copolymer-based Synthesized derivative of Cysteine-loaded nanocarriers from coprecipitation method (Kayal \& Ramanujan, 2010; Kharati et al., 2018; Vafajoo et al., 2018) at constant temperature followed by evaporation of an organic solvent. In addition, the biodegradable and biocompatible polycaprolactone-based copolymer (PCL) were used as a first attempt to encapsulate Synthesized derivative of Cysteine in the Polymeric nanocarrier to produce the polymerbased nanocarrier, and in this work, the biocompatibility of nanocarrier and neuroprotective potential of the encapsulated Synthesized derivative of Cysteine were evaluated in SH-SY5Y cells and MTT reduction for a first time. 


\section{Materials and methods}

\section{Materials}

3-[4,5-dimethylthiazol-2-yl]-2,5-diphenyltetrazolium bromide (MTT), Fetal Bovine Serum (FBS), SH-SY5Y cell line human (Neuroblast from neuronal tissue), Poly(ethylene glycol)-block-poly( $\varepsilon$-caprolactone) methyl ether - PCL-b-PEG (PCL average Mw $\approx 13,000$ $\mathrm{g} / \mathrm{mol}, \mathrm{PEG}$ average $\mathrm{Mw} \approx 5,000 \mathrm{~g} / \mathrm{mol}$ ), Penicillin-Streptomycin solution $(10,000$ unit Penicillin and $10 \mathrm{mg}$ Streptomycin/mL); Trypsin-EDTA solution (0.5 g porcine trypsin and 0.2 g EDTA); $N$-acetylcysteine (Grade $\geq 99 \%$ ); Staurosporine from Streptomyces sp.; Z-VAD-FMK; Lactate Dehydrogenase Activity Assay Kit (LDH) and Dulbecco's modified Eagle medium (DMEM) were purchased from Sigma-Aldrich Chemie GmbH, Germany.

\section{Synthesis of PMI-CYSTEINE}

The PMI-CYSTEINE was synthesized according the recently published paper (Kozma et al., 2018). Briefly, 3,4,9,10-Perylene tetracarboxylic acid dianhydride (PDA) (0.5 mmol), $L$-CYSTEINE (1.05 mmol) and $1 \mathrm{~g}$ of imidazole were added into a Schlenk and heated at $120{ }^{\circ} \mathrm{C}$ for $4 \mathrm{~h}$, while the colour turned from red to violet. The reaction mixture was filtered to eliminate the unreacted PDA, then was cooled, poured into water, and filtered. The recovered solid precipitate is PMI-Cys (15\% yield).

\section{Nanocarriers' preparation}

Synthesized derivative of Cysteine-loaded Polymeric nanocarriers was prepared from coprecipitation method at constant temperature. The NCs were prepared by dissolving PMI-CYSTEINE (5 mg) in $2 \mathrm{~mL}$ of methanol/water (5/1), the PEG-b-PCL, in the determined amounts, was added to the same solution and sonicated for $3 \mathrm{~min}$ and, then, the solution was added drop wise to $10 \mathrm{~mL}$ water during $25 \mathrm{~min}$. stirring was continued for $3 \mathrm{~h}$ to allow complete evaporation of the solvent. After that and in order to remove polymer aggregates, the NCs were filtered by a micro-filter with pore size of $1.5 \mu \mathrm{m}$ (Whatman ${ }^{\circledR}$ GD/X syringe filters) and the obtained suspension was centrifuged at $12,000 \mathrm{rpm}$ for $40 \mathrm{~min}$. Afterwards, the supernatant was alienated and the free PMICYSTEINE which remained in the supernatant was measured using HPLC method. The collected sediment was suspended in $1 \mathrm{~mL}$ deionized water and lyophilized using freeze-drier.

\section{Loading efficiency and capacity}

The loading efficiency (LE) and capacity (LC) of the NCs were determined by HPLC method as mentioned in preparing the NCs and calculated as follow:

$$
\begin{aligned}
& L E(\%)=\frac{\text { Total amount of } \mathrm{PMI}-\text { Cysteine added }- \text { Free SAC }}{\text { Total amount of SAC added }} \times 100 \\
& L C(\%)=\frac{\text { Total amount of } \mathrm{PMI}-\text { Cysteine added }- \text { Free SAC }}{\text { Weight of nanoparticles }} \times 100
\end{aligned}
$$




\section{Nanocarrier' zeta potential determination}

Zeta potential is one of the major physicochemical properties of NCs that determine the stability of NCs in aqueous medium. The zeta potential of nanocarriers was measured in water suspension by Laser Doppler Electrophoresis (LDE) technique using NANOPHOX Particle Size Analysis (Sympatec GmbH, Germany).

\section{Nanocarrier' size analysis}

NCs size distribution were investigated by dynamic light scattering (DLS) technique NANOPHOX Particle Size Analysis (Sympatec GmbH, Germany).

\section{In vitro SH-SY5Y cell culture}

SH-SY5Y cells from the cell line human neuroblast from neural tissue were grown in DMEM (with 10\% Fetal Bovine Serum (FBS) and 0.1\% Pen Strep solution). The cells were kept at $36^{\circ} \mathrm{C}\left(95 \%\right.$ air and $\left.5 \% \mathrm{CO}_{2}\right)$. The cells were Trypsin-EDTA and seeded at a density of $6 \times 10^{4}$ into 96 -well plates. In addition, the cells were evaluated by LUNATM Automatic Cell Counter (Logos Biosystems).

\section{Cell treatment}

Initially, the cells were treated for $24 \mathrm{~h}$ with $20 \mu \mathrm{l}$ of NCs suspension at different specific dilutions that were discussed in the discussion part. To proceed further investigation on neuroprotective potential, SH-SY5Y cells were co-treated with PMI-CYSTEINEcontaining NCs at specific different dilutions and the polymer-cysteine $(20 \mu \mathrm{M})$ alone and staurosporine (St) for comparison. Empty PEG-b-PCL NCs at dilution 1:4 and Nacetylcysteine antioxidant and pan-caspase inhibitor, Z-VAD-fmk (25 $\mu \mathrm{M})$ were applied. First, $10 \mathrm{mM}$ of PMI-CYSTEINE was dissolved in distilled water and stored at $-20^{\circ} \mathrm{C} .10$ $\mathrm{mM}$ of staurosporine and $5 \mathrm{mM}$ of Z-VAD-fmk solutions was prepared in a polar aprotic solvent such as DMSO. Final dilutions of St $(15 \mu \mathrm{M})$, zVAD-fmk (1 mM) and PMICYSTEINE (1 mM) were prepared in distilled water. The final chemical mixtures were present in SH-SY5Y cell cultures $(1 \%$ concentration for staurosporine and PMICYSTEINE, and $10 \%$ for NCs).

\section{Cytotoxicity assay}

SH-SY5Y cells were chosen due to the fact that our purpose was to investigate the PMICYSTEINE-loaded nanocarriers in neuropharmacology. This cell line which has been derived from a bone marrow biopsy of a neuroblastoma patient has been widely used in neurodegenerative and cytotoxic processes (Lopes et al., 2010). To increase the authenticity and accuracy of the evaluation of the nanocarriers cytotoxicity, both MTT reduction and LDH release assays have been used to determine in vitro cell viability.

The results of cytotoxicity test are summarized in Figure 2 and Figure 3. Cell viability and cell death assessments were investigated using LDH and MTT assays. Average concentration of each NCs, which were tested, was about $2 \times 10^{10}$ particles $/ \mathrm{mL}$ corresponding to about $5.5 \times 10^{5}$ particles/cell. Both LDH and MTT assays were done after $24 \mathrm{~h}$ treatment with the specific agents. 


\section{Investigation of Cell viability}

This assay in neuroprotection and biocompatibility studies was investigated by MTT assay. The exact procedure was described by (Liu et al., 1997). In sum, the cells were incubated with $0.25 \mathrm{mg} / \mathrm{mL}$ MTT for $4 \mathrm{~h}$ at $37^{\circ} \mathrm{C}$ and the reaction was stopped by adding a solution of $50 \% \mathrm{DMF}$ and $20 \%$ SDS at pH 4.8 . After $24 \mathrm{~h}$, the amount of formazan product was determined by measuring the absorbance with a 96 -well plate reader at a test wavelength of $570 \mathrm{~nm}$ and reference wavelength of $655 \mathrm{~nm}$.

\section{Statistical analysis}

All of the data after normalization, were analyzed using Statistica software (StatSoft Inc., Tulsa, OK, USA).

\section{Results and discussion}

\section{Preparation of Polymeric nanocarriers}

In this work, the Polymeric NCs (PCL-b-PEG) were prepared from coprecipitation method (Kayal \& Ramanujan, 2010) at constant temperature. The rate of solution addition to water was examined and the optimize rate was about $0.15 \mathrm{~mL} / \mathrm{min}$. NCs with about $2 \mathrm{mg} / \mathrm{mlL}$ PEG-b-PCL (in the solution) were selected for this study because of the lowest polydispersity $(\mathrm{PdI}<0.2)$. Eventually during the evaporation of the organic solvent the Polymeric NCs were formed.

The average size of the PEG-b-PCL NCs without PMI-CYSTEINE which has been measured by DLS was about $108 \mathrm{~nm}$ with the $\mathrm{PdI}<0.2$. The zeta potential of Polymeric NCs was $-17 \mathrm{mV}$. The stability of NCs dispersion was investigated over 5 weeks too and approved the results.

\section{Preparation of PMI-CYSTEINE loaded nanocarriers}

The brief procedure of PEG-b-PCL PMI-CYSTEINE-loaded NCs preparation was described above. As a matter of fact, polymers as well as PMI-CYSTEINE $(0.5 \mathrm{mg} / \mathrm{mL})$ were dissolved in methanol/water with the Copolymer/drug ratio of 5:1. The average size of the PMI-CYSTEINE-loaded NCs that were measured by DLS was about $126 \mathrm{~nm}$ (Figure 2) and the polydispersity (PdI) index of PMI-CYSTEINE-loaded NC was $<0.2$. The zeta potential of PMI-CYSTEINE-loaded PEG-b-PCL NCs was $-11 \mathrm{mV}$. Also the loading efficiency of PMI-CYSTEINE into NC was examined and the result shows $81 \%$ with the entrapment efficiency of about $93 \%$. In addition, the PMI-CYSTEINE release at $24 \mathrm{~h}$ was $46.9 \%$ with the correlation coefficients of $0.8520,0.9492$ and 0.6926 for zero order, Higuchi and Hixson-Crowell, respectively. The stability of PMI-CYSTEINE-loaded NCs was examined for 5 weeks which was verified by repeated measurements of their zeta potential. 

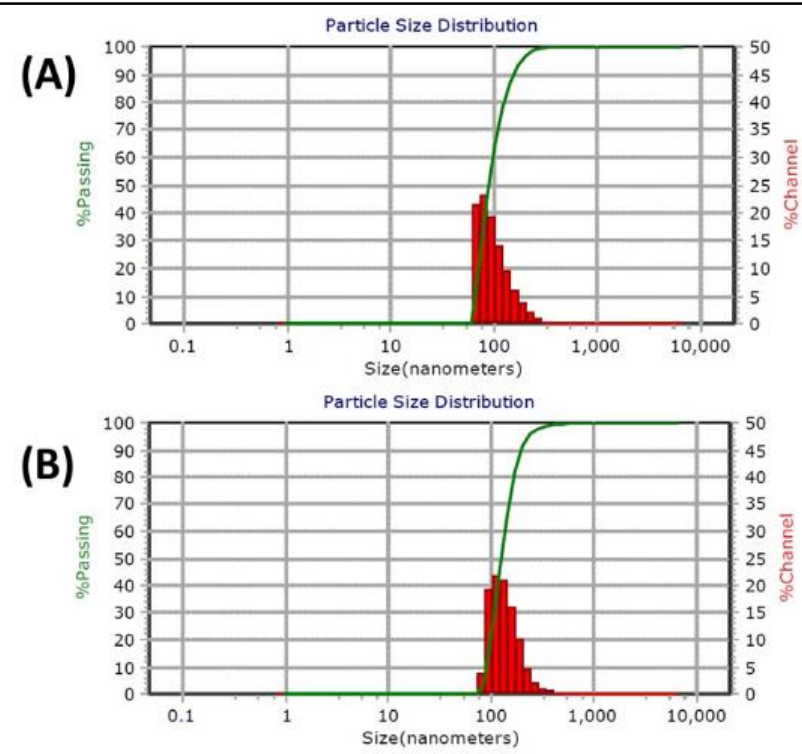

Figure 2. Size distribution of the polymeric NC and Synthesized derivative of Cysteine loaded polymeric NC measured by DLS technique (A) and (B) respectively.

The detailed characterization of NCs are shown in Table 1.

Table 1. The composition and detailed characterization of NCs

\begin{tabular}{cccc}
\hline Sample & The average size (nm) & PdI & Zeta Potential (mV) \\
\hline PEG-b-PCL/empty & 89 & 0.161 & -5 \\
PEG-b-PCL/PMI-CYSTEINE & 126 & 0.188 & -11 \\
\hline
\end{tabular}

\section{Investigation of Biocompatibility}

After 24 hours, treatment evoked significant toxicity (2-4.5-fold increases in LDH level) as depicted in Figure 3 as well decrease in cell viability, and 18\% decrease in MTT assay, as shown in Figure 4.

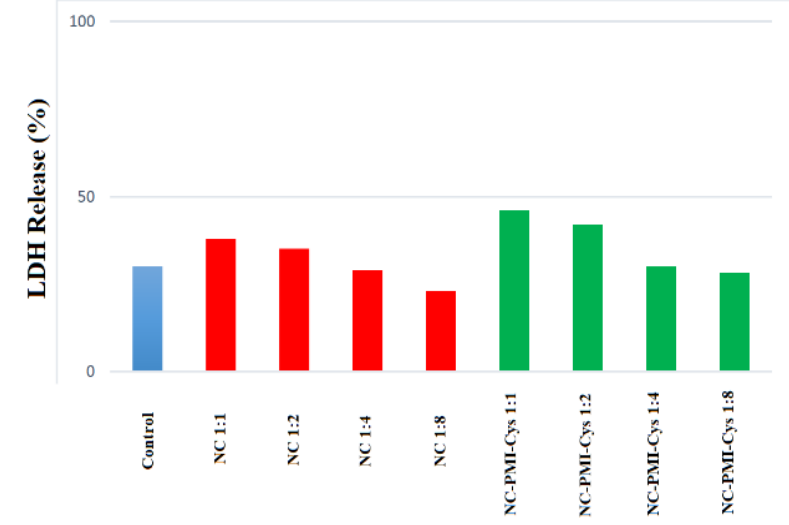

Figure 3. LDH Release of empty and PMI-CYSTEINE containing polymeric NCs, (one way ANOVA followed by Tukey's test ( $\mathrm{P}<0.001$ vs. vehicle-treated cells; and $\mathrm{P}<0.001 \mathrm{~N}-1$ vs. PMI-CYSTEINEtreated cells)). 


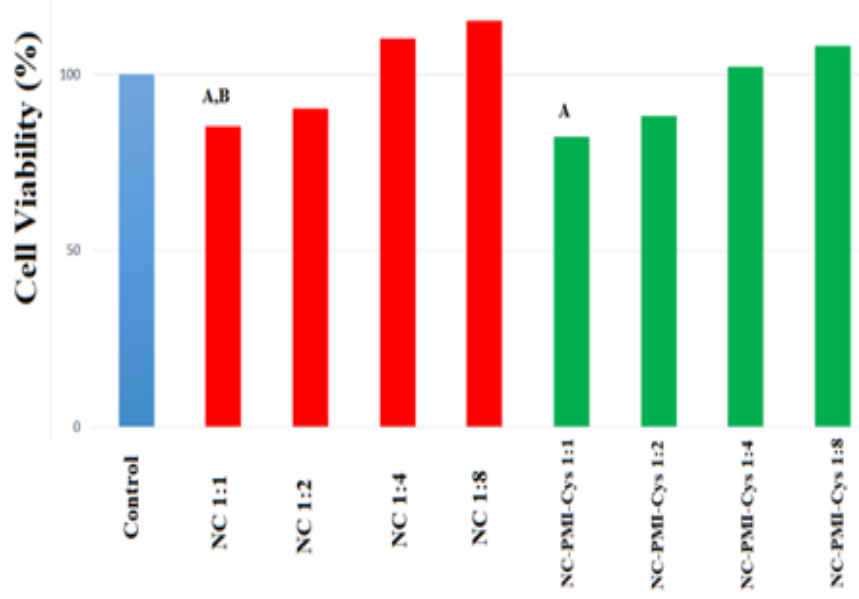

Figure 4. Cell viability (MTT Reduction assay) of empty and PMI-CYSTEINE containing polymeric NCs, (one way ANOVA followed by Tukey's test (A : P < 0.001 vs. vehicle-treated cells; B : P $<0.001$ N-1 vs. PMI-CYSTEINE-treated cells))

In this case, Polymeric NCs at dilution 1:1 have a slightly more toxic effect in both used assays for PMI-CYSTEINE-loaded NCs to compare with empty copolymer. As it has been depicted in Figure 3, the cytotoxic effect was decreased by increasing the dilutions of NCs, so the concentration of Polymeric NCs below $1 \times 10^{10}$ particles $/ \mathrm{ml}$ can be deliberated as a safe concentration for the cell line.

\section{Neuroprotection studies}

Initially, researcher's introduced the programmed cell death to describe ostensibly predetermined pattern in which specific cells die during a procedure, which has a crucial role in the development of the nervous system (Ito et al., 2003; Ueda et al., 2002; Zalucki et al., 2017). There is another mechanism to describe neuronal cell loss under different neurodegenerative conditions in which apoptotic processes and also oxidative stress have a decisive role (Bredesen et al., 2006). Since the therapeutic effect of PMICYSTEINE was determined in various models of neurodegenerative disease, such as Alzheimer, (Javed et al., 2011) and of course the antioxidant effect and related mechanism were confirmed, (Colín-González et al., 2012; Niedzielska et al., 2016), we have used the model of pro-apoptotic factor staurosporine-evoked cell damage in SHSY5Y cell line. This effort was to tackle on the issue of neuroprotective potential of PMI CYSTEINE loaded NCs in comparison to the effect of PMI-CYSTEINE empty to have steps coming along with the recent research. The result of MTT reduction assay showed about $18 \%$ neuroprotective effect of Z-VAD-fmk as model inhibitor and about $12 \%$ neuroprotective effect of PMI-CYSTEINE against St-evoked SH-SY5Y cell damage which has been depicted in Figure 5. In addition, 22\% protection for PMI-CYSTEINE loaded Polymeric NC at dilution 1:8 in St Model of cell damage is clear and the amazing result is the PMI-CYSTEINE loaded Polymeric NC at any dilution has not shown more toxicity than staurosporine. 


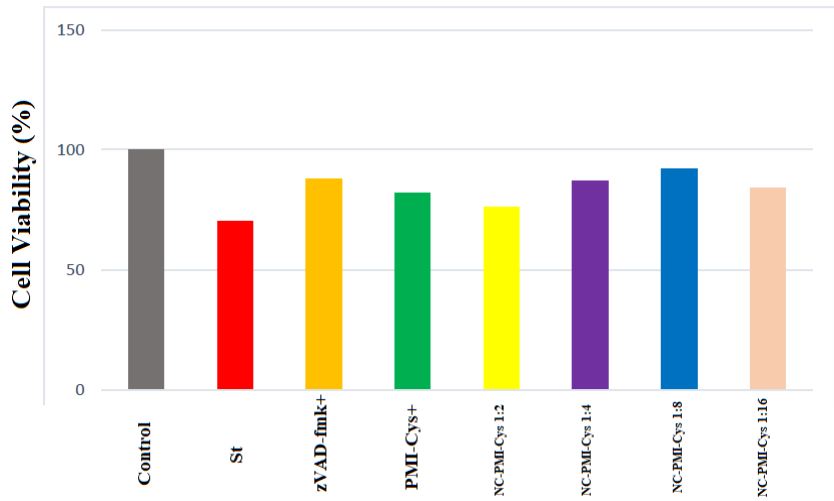

Figure 5. Neuroprotective effects of polymeric NCs containing PMI-CYSTEINE against staurosporine (St). (one way ANOVA followed by Tukey's test ( $<<0.001$ vs. vehicle-treated cells; $\mathrm{P}<0.001$ vs. Sttrated cells))

A striking result which could be the PMI-CYSTEINE encapsulated into Polymeric NC compared with free PMI-CYSTEINE would shows 10\% more neuroprotective effect and it means the PMI-CYSTEINE encapsulated into Polymeric NC compared with free PMICYSTEINE can gives considerable results.

\section{Conclusion}

The polymeric NC has been prepared from coprecipitation method at constant temperature followed by evaporation of an organic part of the solvent. This method was applied to investigate the neuroprotective action of PMI-CYSTEINE, which is encapsulated into polymeric NC. This work is the first report to encapsulate PMI-CYSTEINE in the polymeric NC representing some critical facts of the neuroprotective action of PMI-CYSTEINE. The results from biocompatibility tests indicated that polymeric NCs at dilution 1:1 have been showing a slightly more toxic effect in both used assays for PMI-CYSTEINE-loaded NCs as compared to the empty one. However, the cytotoxic effect was decreased by increasing the dilutions of NCs, so it was optimized to the concentration of Polymeric NCs which could be mentioned as a safe concentration for the cell line. The results from Neuroprotection studies indicated that PMI-CYSTEINE was encapsulated into Polymeric NCs as compared to the free PMI-CYSTEINE which would show more neuroprotective effect and give the same neuroprotective effect as a free PMI-CYSTEINE at lower concentration. Thus, the MTT assay showed that PMI-CYSTEINE loaded in PEG-b-PCL NCs has a significant neuroprotective potential against Z-VAD-fmk and St-evoked SH-SY5Y cell damage. This work represented important results which may be considered by more advanced researches. In fact, in future, we want to focus on the in vivo strategies and optimizations based on the stability, tissue interactions and reducing the side effects.

\section{References}

Bredesen, DE, Rao, RV, Mehlen, P. (2006). Cell death in the nervous system. Nature, 443(7113):796.

Carragher, NO. (2006). Calpain inhibition: a therapeutic strategy targeting multiple disease states. Curr. Pharmaceut. design, 12(5):615-638. 
Chen, G, Roy, I, Yang, C, Prasad, PN. (2016). Nanochemistry and nanomedicine for nanoparticle-based diagnostics and therapy. Chem. Rev., 116(5):2826-2885.

Colín-González, AL, Santana, RA, Silva-Islas, CA, Chánez-Cárdenas, ME, Santamaría, A, Maldonado, PD. (2012). The antioxidant mechanisms underlying the aged garlic extract-and S-allylcysteine-induced protection. Oxidat. Med. Cell. Longev., 2012. http://dx.doi.org/10.1155/2012/907162

Deljoo, S, Rabiee, N, Rabiee, M. (2019). Curcumin-hybrid nanoparticles in drug delivery system. Asian J. Nanosci. Mater., 2(1):66-91.

Ghasemi, A, Rabiee, N, Ahmadi, S, Hashemzadeh, S, Lolasi, F, Borzogomid, M, Kalbasi, A, Nasseri, B, Shiralizadeh Dezfuli, A, Aref, AR, Karimi, M, Hamblin, MR. (2018). Optical Assays Based on Colloidal Inorganic Nanoparticles. Analyst., 143:3249-3283.

Imai, T, Kosuge, Y, Endo-Umeda, K, Miyagishi, H, Ishige, K, Makishima, M, Ito, Y. (2014). Protective effect of $\mathrm{S}$-allyl-l-cysteine against endoplasmic reticulum stress-induced neuronal death is mediated by inhibition of calpain. Amin. Acid., 46(2):385-393.

Ito, Y, Kosuge, Y, Sakikubo, T, Horie, K, Ishikawa, N, Obokata, N, Yokoyama, E, Yamashina, K, Yamamoto, M, Saito, H, Arakawa, M, Ishige, K. (2003). Protective effect of S-allyl-L-cysteine, a garlic compound, on amyloid $\beta$-protein-induced cell death in nerve growth factordifferentiated PC12 cells. Neurosci. Res., 46(1):119-125.

Javed, H, Khan, MM, Khan, A, Vaibhav, K, Ahmad, A, Khuwaja, G, Ahmed, ME, Raza, SS, Ashafaq, M, Tabassum, R, Siddiqui, MS, El-Agnaf, OM, Safhi, MM, Islam, F. (2011). S-allyl cysteine attenuates oxidative stress associated cognitive impairment and neurodegeneration in mouse model of streptozotocin-induced experimental dementia of Alzheimer's type. Brain Res., 1389:133-142.

Karimi, M, Mansouri, MR, Rabiee, N, Hamblin, MR. (2018 a). Advances in Nanomaterials for Drug Delivery: Polymeric, Nanocarbon, and Bio-inspired: Morgan \& Claypool Publishers.

Karimi, M, Mansouri, MR, Rabiee, N, Hamblin, MR. (2018 b). Advances in nature-inspired nanomaterials. Advances in Nanomaterials for Drug Delivery: Polymeric, Nanocarbon, and Bio-inspired.

Kayal, S, Ramanujan, R. (2010). Doxorubicin loaded PVA coated iron oxide nanoparticles for targeted drug delivery. Mater. Sci. Eng. C, 30(3):484-490.

Kharati, M, Foroutanparsa, S, Rabiee, M, Salarian, R, Rabiee, N, Rabiee, G. (2018). Early diagnosis of multiple sclerosis based on optical and electrochemical biosensors: comprehensive perspective Curr. Anal. Chem., 14:1-11.

Kodai, S, Takemura, S, Minamiyama, Y, Hai, S, Yamamoto, S, Kubo, S, Yoshida, Y, Niki, E, Okada, S, Hirohashi, K, Suehiro, S. (2007). S-allyl cysteine prevents CCl4-induced acute liver injury in rats. Free Radical Res., 41(4):489-497. 
Kozma, E, Galeotti, F, Grisci, G, Barba, L, Arrighetti, G, Catellani, M, Scavia, G, Porzio, W. (2018). Perylene diimide cysteine derivatives self-assembled onto (111) gold surface: Evidence of ordered aggregation. Surf. Sci., 675:15-25.

Liu, Y, Peterson, DA, Kimura, H, Schubert, D. (1997). Mechanism of cellular 3 - (4, 5 - dimethylthiazol - 2 - yl) - 2, 5 -diphenyltetrazolium bromide (MTT) reduction. J. Neurochem., 69(2):581-593.

Lopes, FM, Schröder, R, da Frota Júnior, MLC, Zanotto-Filho, A, Müller, CB, Pires, AS, Meurer, RT, Colpo, GD, Gelain, DP, Kapczinski, F, Moreira, JC, Fernandes, Mda, C, Klamt, F. (2010). Comparison between proliferative and neuron-like SH-SY5Y cells as an in vitro model for Parkinson disease studies. Brain Res., 1337:85-94.

Moriguchi, T, Matsuura, H, Kodera, Y, Itakura, Y, Katsuki, H, Saito, H, Nishiyama, N. (1997). Neurotrophic activity of organosulfur compounds having a thioallyl group on cultured rat hippocampal neurons. Neurochem. Res., 22(12):1449-1452.

Nasseri, B, Soleimani, N, Rabiee, N, Kalbasi, A, Karimi, M, Hamblin, MR. (2018). Point-of-care microfluidic devices for pathogen detection. Biosens. Bioelectron., 117:112-128.

Niedzielska, E, Smaga, I, Gawlik, M, Moniczewski, A, Stankowicz, P, Pera, J, Filip, M. (2016). Oxidative stress in neurodegenerative diseases. Molecular neurobiol., 53(6):4094-4125.

Rabiee, M, Rabiee, N, Salarian, R, \& Rabiee, G. (2019a). Bioactive nanomaterials. Introduction to Nanomaterials in Medicine.

Rabiee, M, Rabiee, N, Salarian, R, Rabiee, G. (2019b). Introduction to Nanomaterials in Medicine: Morgan \& Claypool Publishers.

Rabiee, M, Rabiee, N, Salarian, R, Rabiee, G. (2019c). Nanomaterials: concepts. Introduction to Nanomaterials in Medicine.

Rabiee, N, Kiani, M, Bagherzadeh, M, Rabiee, M, Ahmadi, S. (2019). Nanoparticle (NP)-Based Delivery Vehicles.

Rabiee, N, Rabiee, M. (2018 a). Biocompatibility and neuroprotective potential of encapsulated S-Allyl-L-Cysteine into PCL-based nanocarrier. Drug Deliv. Lett., 8(3):242-247.

Rabiee, N, Rabiee, M. (2018 b). Magnetic Stimuli-Responsive Cobalt Ferrite Nanoparticle as Theranostic agents for Targeted Delivery. Curr. Nanomater., 3(3):160-167.

Rabiee, N, Rabiee, M. (2019). A promising Stimuli-Responsive nanocomposite as a Theranostic agent for Targeted Delivery. J. Bioengin. Res., 1(1):27-36.

Svenson, S. (2012). Clinical translation of nanomedicines. Curr. Opin. Solid State Mater. Sci., 16(6):287-294.

Szczęch, M, Szczepanowicz, K, Jantas, D, Piotrowski, M, Kida, A, Lasoń, W, Warszyński, P. (2017). Neuroprotective action of undecylenic acid (UDA) encapsulated into PCL nanocarriers. Colloids Surf. Physicochem. Eng. Aspects, 532:41-47. 
Thomson, M, Ali, M. (2003). Garlic [Allium sativum]: a review of its potential use as an anticancer agent. Curr. cancer drug targets, 3(1):67-81.

Ueda, S, Masutani, H, Nakamura, H, Tanaka, T, Ueno, M, Yodoi, J. (2002). Redox control of cell death. Antioxid. Redox Signal., 4(3):405-414.

Uttara, B, Singh, AV, Zamboni, P, Mahajan, R. (2009). Oxidative stress and neurodegenerative diseases: a review of upstream and downstream antioxidant therapeutic options. Curr. neuropharmacol., 7(1):65-74.

Vafajoo, A, Rostami, A, Parsa, SF, Salarian, R, Rabiee, N, Rabiee, G, Tahriri, M, Yadegari, A, Vashaee, D, Tayebi, L, Hamblin, MR. (2018). Early Diagnosis of Disease Using Microbead Array Technology: A Review. Anal. Chim. Acta., 1032:1-17

Vafajoo, A, Salarian, R, Rabiee, N. (2018). Biofunctionalized microbead arrays for early diagnosis of breast cancer. Biomed. Phys. Eng. Exp., 4(6):065028.

Zalucki, M, Zalucki, J, Perkins, L, Schramm, K, Vassão, D, Gershenzon, J, Heckel, DG. (2017). A Generalist Herbivore Copes with Specialized Plant Defence: the Effects of Induction and Feeding by Helicoverpa armigera (Lepidoptera: Noctuidae) Larvae on Intact Arabidopsis thaliana (Brassicales) Plants. J. chem. ecology, 43(6):608-616.

How to cite this article: Ehab M. Ali, Ahmed Solaiman Hamed. Bioorganic Investigation of ENCapsulated Cysteine Derivative into Polymeric Nanocarrier. International Journal of Advanced Biological and Biomedical Research, 2019, 7(4), 342-352. Link: http://www.ijabbr.com/article 35651.html 